УДК 512.554.3

\title{
The Highest Dimension of Commutative Subalgebras in Chevalley Algebras
}

\section{Galina S. Suleimanova*}

Khakas Technical Institute Branch of Siberian Federal University

Shchetinkin, 27, Abakan, 665017 Russia

Received 17.12.2018, received in revised form 20.01.2019, accepted 20.02.2019

Let $L_{\Phi}(K)$ denotes a Chevalley algebra with the root system $\Phi$ over a field K. In 1945 A. I. Mal'cev investigated the problem of describing abelian subgroups of highest dimension in complex simple Lie groups. He solved this problem by transition to complex Lie algebras and by reduction to the problem of describing commutative subalgebras of highest dimension in the niltriangular subalgebra. Later these methods were modified and applied for the problem of describing large abelian subgroups in finite Chevalley groups. The main result of this article allows to calculate the highest dimension of commutative subalgebras in a Chevalley algebra $L_{\Phi}(K)$ over an arbitrary field.

Keywords: Chevalley algebra, commutative subalgebra.

DOI: 10.17516/1997-1397-2019-12-3-351-354.

\section{Introduction}

Let $L_{\Phi}(K)$ denotes a Chevalley algebra with the root system $\Phi$ over a field $K$, and let $\Pi$ be a fundamental system of roots. The elements $\left\{e_{r}, h_{p} \mid r \in \Phi, p \in \Pi\right\}$ form a basis of $L_{\Phi}(K)$, called a Chevalley basis [1]. Let $N \Phi(K)$ denotes a niltriangular subalgebra in $L_{\Phi}(K)$ with the basis $\left\{e_{r} \mid r \in \Phi^{+}\right\}$.

In 1945 A. I. Mal'cev investigated the problem of describing abelian subgroups of highest dimension in complex simple Lie groups [7]. He solved this problem by transition to complex Lie algebras and by reduction to the problem of describing commutative subalgebras of highest dimension in the niltriangular subalgebra $N \Phi(\mathbb{C})$.

Later these methods were modified and applied for the problem of describing large abelian subgroups in finite Chevalley groups $[3-5,8,9]$. Given a group-theoretic property $\mathcal{P}$, we recall that every $\mathcal{P}$-subgroup of largest order in a finite group is a large $\mathcal{P}$-subgroup.

The generalization of Mal'cev problem [7] for Chevalley algebras $L_{\Phi}(K)$ over an arbitrary field $K$ was pointed in [6].

The main result of this article is Theorem 1, which allows to calculate the highest dimension of commutative subalgebras in a Chevalley algebra $L_{\Phi}(K)$ over an arbitrary field.

*suleymanova@list.ru

(C) Siberian Federal University. All rights reserved 


\section{Preliminary remarks and notation}

Let $L_{\Phi}(K)$ denotes a Chevalley algebra with the root system $\Phi$ over a field $K$, and let $\Pi$ be a fundamental system of roots. The elements $\left\{e_{r}, h_{p} \mid r \in \Phi, p \in \Pi\right\}$ form a basis of $L_{\Phi}(K)$, called a Chevalley basis. The elements of this basis multiply together as follows:

$$
\begin{array}{ll}
h_{r} * h_{s}=0, & r, s \in \Pi, \\
h_{r} * e_{s}=A_{r s} e_{s}, & r \in \Pi, s \in \Phi, \\
e_{r} * e_{-r}=h_{r}, & r \in \Phi, \\
e_{r} * e_{s}=0, & r, s \in \Phi, r+s \notin \Phi, \\
e_{r} * e_{s}=N_{r s} e_{r+s}, &
\end{array}
$$

where the elements $N_{r s}$ are called the structure constants of $L_{\Phi}(K)$, and $A_{r s}=\frac{2(r, s)}{(r, r)}$. The elements $\left\{h_{p} \mid p \in \Pi\right\}$ form a basis for a Cartan subalgebra $H$ [1].

A subset $\Psi$ of the root system $\Phi$ is called a commutative, if $r+s \notin \Phi$ for all $r, s \in \Psi$ [7]. A subset $\Psi$ of the root system $\Phi$ is said to be $p$-commutative, if in the algebra $N \Phi(K)$ over a field $K$ of characteristic $p$ we have $e_{r} * e_{s}=0$ for all $r, s \in \Psi$ [8].

Further, we use a regular ordering of roots [1, Lemma 5.3.1]. Let $x \in L_{\Phi}(K)$ and

$$
x=a_{1} e_{r_{1}}+a_{2} e_{r_{2}}+\cdots+h+c_{1} e_{s_{1}}+c_{2} e_{s_{2}}+\ldots,
$$

where $r_{i} \in \Phi^{-}, s_{i} \in \Phi^{+}, r_{1}<r_{2}<\ldots, s_{1}<s_{2}<\ldots, h=b_{1} h_{p_{1}}+b_{2} h_{p_{2}}+\ldots, p_{1}, p_{2}, \cdots \in \Pi$. We consider the first non-zero term in (1). If this term has the form $t e_{r}, r \in \Phi$, then we denote $b(x)=e_{r}$, else we denote $b(x)=h$. For $M \subseteq L$ we set $b(M)=\{b(x) \mid x \in M\}$.

Lemma 1. If $x * y=0$ for $x, y \in L_{\Phi}(K)$, then $b(x) * b(y)=0$.

Proof. Let

$$
\begin{aligned}
& x=a_{1} e_{r_{1}}+a_{2} e_{r_{2}}+\cdots+h+c_{1} e_{s_{1}}+c_{2} e_{s_{2}}+\ldots, \\
& y=a_{1}^{\prime} e_{r_{1}^{\prime}}+a_{2}^{\prime} e_{r_{2}^{\prime}}+\cdots+h^{\prime}+c_{1}^{\prime} e_{s_{1}^{\prime}}+c_{2}^{\prime} e_{s_{2}^{\prime}}+\ldots,
\end{aligned}
$$

where $r_{1}+r_{1}^{\prime} \in \Phi^{-}, r_{1}<r_{2}<\cdots<s_{1}<s_{2}<\ldots, r_{1}^{\prime}<r_{2}^{\prime}<\cdots<s_{1}^{\prime}<s_{2}^{\prime}<\ldots$ Then in the expression of $x * y$ as a linear combination of $e_{r}, r \in \Phi$, every $e_{r}\left(r \in \Phi^{-}\right)$has the form $e_{r_{i}+r_{i}^{\prime}}, e_{r_{i}+s_{i}^{\prime}}, e_{s_{i}+r_{i}^{\prime}}, e_{r_{i}}$ or $e_{r_{i}^{\prime}}$. Since $r_{i}-r_{1} \in V^{+}, r_{i}^{\prime}-r_{1}^{\prime} \in V^{+}(i \neq 1)$, where $V^{+}$is a certain positive subspace, then $\left(r_{i}+r_{i}^{\prime}\right)-\left(r_{1}+r_{1}^{\prime}\right)=\left(r_{i}-r_{1}\right)+\left(r_{i}^{\prime}-r_{1}^{\prime}\right) \in V^{+}$, so $r_{1}+r_{1}^{\prime}<r_{i}+r_{i}^{\prime}$. Since $h t\left(r_{1}+r_{1}^{\prime}\right)<h t\left(r_{i}\right)$ and $h t\left(r_{1}+r_{1}^{\prime}\right)<h t\left(r_{i}^{\prime}\right)$, we have $r_{1}+r_{1}^{\prime}<r_{i}$ and $r_{1}+r_{1}^{\prime}<r_{i}^{\prime}$. Hence $b(x * y)=e_{r_{1}+r_{1}^{\prime}}$, so $x * y \neq 0$, that gives a contradiction. Analogously, the remaining cases when $b(x), b(y), b(x) * b(y)$ are not in $H$ give a contradiction.

Let $b(x)=h, b(y)=a_{1}^{\prime} e_{r_{1}^{\prime}}$ and $h * a_{1}^{\prime} e_{r_{1}^{\prime}} \neq 0$. Then $b(x * y)=e_{r_{1}^{\prime}}$, so again $x * y \neq 0$.

In the case

$$
\begin{gathered}
x=c_{1} e_{s_{1}}+c_{2} e_{s_{2}}+\ldots, \\
y=a_{1}^{\prime} e_{r_{1}^{\prime}}+a_{2}^{\prime} e_{r_{2}^{\prime}}+\cdots+h^{\prime}+c_{1}^{\prime} e_{s_{1}^{\prime}}+c_{2}^{\prime} e_{s_{2}^{\prime}}+\ldots,
\end{gathered}
$$

where $r_{1}^{\prime}=-s_{1}$, the expression of $x * y$ contains a term $h_{r_{1}^{\prime}}$. If $x * y=0$, then in the expression of $x$ and $y$ there exists another pair of terms of form $e_{s_{i}}, e_{-s_{i}}$, respectively. Hence $s_{1}<s_{i}$ and $-s_{1}<-s_{i}$, a contradiction. This completes the proof of Lemma 1.

Note that in the case $b(M)=\left\{e_{r} \mid r \in \Phi^{+}\right\}$the set of corresponding roots $r \in \Phi^{+}$coincides with the set $\mathcal{L}_{1}(M)([2,4])$. 


\section{The highest dimension of commutative subalgebras}

Theorem 1. Let $L_{\Phi}(K)$ be a Chevalley algebra with the root system $\Phi$ over an arbitrary field $K$ of characteristic $p$. Let $m$ be a maximal order of $p$-commutative subsets of roots in $\Phi$, and let $k$ be a dimension of the center of $L_{\Phi}(K)$. The highest dimension of commutative subalgebras of $L_{\Phi}(K)$ equals $m+k$.

Lemma 2. Let $A$ be a commutative subalgebra of $L=L_{\Phi}(K)$. Then there exists an automorphism of $L$ transforming $A$ to a subalgebra $B$ in $L$ such that for all $x \in B$ either $b(x)=e_{r}$, $r \in \Phi^{-}$, or $b(x)=h \in H$, where $h$ is an element of the center of $L$.

Proof. Let $x_{r}(t)$ denotes an automorphism of a Chevalley algebra, effecting on the elements of a Chevalley basis as follows:

$$
\begin{gathered}
e_{r} \rightarrow e_{r}, \\
h_{s} \rightarrow h_{s}-t A_{s r} e_{r}(s \in \Pi), \\
e_{-r} \rightarrow e_{-r}+t h_{r}-t^{2} e_{r}, \\
e_{s} \rightarrow \sum_{i=0}^{q} M_{r, s, i} t^{i} e_{i r+s}(s \in \Phi \backslash\{ \pm r\}), \quad M_{r, s, 0}:=1, \\
M_{r, s, i}:= \pm(p(r, s)+i / / i) .
\end{gathered}
$$

Let

$$
x=h+c_{1} e_{s_{1}}+c_{2} e_{s_{2}}+\ldots
$$

Suppose that there exists a fundamental root $p$ such that $h * e_{p} \neq 0$ and $s_{1} \neq p$. Since

$$
h * e_{p}=\left(b_{1} h_{p_{1}}+b_{2} h_{p_{2}}+\ldots\right) * e_{p}=\left(b_{1} A_{p_{1}, p}+b_{2} A_{p_{2}, p}+\ldots\right) e_{p},
$$

then the automorphism $x_{-p}(1)$ transforms $h$ to

$$
h+\left(b_{1} A_{p_{1}, p}+b_{2} A_{p_{2}, p}+\ldots\right) e_{p},
$$

where $b_{1} A_{p_{1}, p}+b_{2} A_{p_{2}, p}+\cdots \neq 0$. Hence $b\left(x_{-p}(1)(x)\right)=e_{-p}$.

If $s_{1}=p$, then first we obtain $c_{1}=0$, up to a certain automorphism $x_{p}(t), p \in \Pi$, and again $b\left(x_{-p}(1)(x)\right)=e_{-p}$.

Suppose that there exists $y \in A$ such that $b(y)=e_{r}, r \in \Phi^{-}$, and $b\left(x_{ \pm p}(1)(y)\right)$ equals $h \in H$ or $e_{r}, r \in \Phi^{+}$. Taking into account the relations (2)-(5), we deduce that $b(y)=e_{-p}$. Since $h * e_{p} \neq 0$ hence $h * e_{-p} \neq 0$. By Lemma 1, this is a contradiction.

If

$$
x=c_{1} e_{s_{1}}+c_{2} e_{s_{2}}+\ldots
$$

then $b\left(x_{-s_{1}}(1)(x)\right)=e_{-s_{1}}$.

Suppose that there exists $y \in A$ such that $b(y)=e_{r}, r \in \Phi^{-}$, and $b\left(x_{-s_{1}}(1)(y)\right.$ equals $h \in H$ or $e_{r}, r \in \Phi^{+}$. Taking into account the relations (2)-(5), we deduce that $b(y)=e_{-s_{1}}$. By Lemma 1, this is a contradiction.

The lemma is proved.

Now the Theorem 1 follows from Lemma 1 and Lemma 2. 


\title{
References
}

[1] R.Carter, Simple groups of Lie type, Wiley and Sons, New York, 1972.

[2] E.A.Kirillova, G.S.Suleimanova, Highest dimension commutative ideals of a niltriangular subalgebra of a Chevalley algebra over a field, Trudy of Inst. of Math. \& Mech. UrO RAN, 24(2018), no. 3, 98-108.

[3] A.S.Kondratiev, Subgroups of finite Chevalley groups, Russian Math. Surveys, 41(1986), no. $1,65-118$.

[4] V.M.Levchuk, G.S.Suleimanova, Extremal and maximal normal abelian subgroups of a maximal unipotent subgroup in groups of Lie type, Journal of Algebra, 349(2012), no.1, $98-116$.

[5] V.M.Levchuk, G.S.Suleimanova, Thompson subgroups and large abelian unipotent subgroups of Lie-type groups, Journal of Siberian Federal University. Mathematics \& Physics, 6(2013), no. 1, 64-74.

[6] V.M.Levchuk, G.S.Suleimanova, The generalized Mal'cev problem on abelian subalgebras of the Chevalley algebras, Lobachevskii Journal of Mathematics, 86(2015), no. 4, 384-388.

[7] A.I.Mal'cev, Commutative subalgebras in semisimple Lie algebras, Izv. Akad. Nauk SSSR. Ser. Mat., 8(1945), 291-300.

[8] E.P.Vdovin, Large Abelian Unipotent Subgroups of Finite Chevalley Groups, Algebra \& Logic, 40(2001), no. 5, 292-305.

[9] E.P.Vdovin, Maximal Orders of Abelian Subgroups in Finite Chevalley Groups, Math. Notes, 69(2001), no. 4, 475-498.

\section{Наивысшая размерность коммутативных подалгебр алгебр Шевалле}

\author{
Галина С. Сулейманова \\ Хакасский технический институт \\ Филиал Сибирского федерального университета \\ Щетинкина, 27, Абакан, 665017 \\ Россия
}

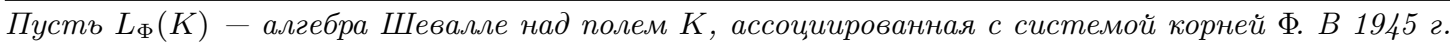
А.И. Мальцев исследовал проблему описания абелевых подгрупп наивысшей размерности в комплексных простых группах Ли. Он решил эту проблему переходом $к$ комплексным алгебрам Ли и редукиией к проблеме описания коммутативных подалгебр наивысшей размерности в нильтреугольной подалгебре. Позже эти методы модифицировались и применялисъ для решения проблемы описания больших абелевых подгрупп конечных групп Шевалле. Основной результат данной статьи позволяет вычислить наивысшую размерность коммутативных подалгебр алгебры Шевалле $L_{\Phi}(K)$ над прочзвольным полем.

Ключевые слова: алгебра Шевалле, коммутативная подалгебра. 